\title{
Cirugía mínimamente invasiva en la incontinencia urinaria femenina: TVT-O
}

\author{
V. Solà Dalenz*, J. Pardo Schanz*, P. Ricci Arriola*, E. Guiloff Fische*, H. Chiang Miranda** \\ *Unidad de Uroginecología y Cirugía Vaginal, Clinica Las Condes. **Departamento de Urología, \\ Clinica Las Condes. Santiago de Chile.
}

Actas Urol Esp 2006; 30 (1): 61-66

\section{RESUMEN}

CIRUGÍA MÍNIMAMENTE INVASIVA EN LA INCONTINENCIA URINARIA FEMENINA: TVT-O

Objetivo: Presentamos nuestra experiencia en la aplicación de TVT-O, en la corrección quirúrgica de la IOE.

Método: Entre abril y diciembre 2004, se realizaron 96 TVT-O a pacientes de la Unidad de Uroginecología y Cirugía Vaginal, del Departamento de Ginecología y Obstetricia de la Clínica Las Condes. Mediana de edad 54 años, peso $65 \mathrm{~kg}$. Test de urodinamia: IOE grado II 80 casos, IOE grado III 4, IOE grado 0 3, e IOM 9. Se utilizó el Sistema Gynecare TVT Obturador de Ethicon, Johnson \& Johnson.

Resultados: Mediana tiempo operatorio TVT-O: 7 minutos. Se asoció alguna cirugía ginecológica en 77. No se presentaron complicaciones durante el acto quirúrgico. No se observó daño de vejiga ni vías urinarias. Durante el postoperatorio inmediato se presentó sólo una complicación (1,04\%) correspondiendo a retención urinaria, por sobrecorrección. Durante el postoperatorio tardío una complicación (1,04\%), exposición de la cinta de polipropileno en la mucosa de la pared vaginal anterior. Se obtuvo solución de la incontinencia de orina de esfuerzo en las 96 pacientes. La mediana de seguimiento 6 meses, 51 casos con seguimiento mayor a 6 meses.

Conclusiones: TVT-O es una técnica quirúrgica para el tratamiento de la IOE, con resultados muy promisorios. Al igual que los otros tipos de TOT, su eficacia deberá ser evaluada a largo plazo.

Palabras clave: Cirugía minimamente invasiva. Incontinencia de orina. TVT-O (tension free vaginal tape obturator). Cirugía ginecológica asociada a TVT-O. Complicaciones asociadas a TVT-O.

\section{ABSTRACT \\ MINIMAL INVASIVE SURGERY IN FEMALE URINARY INCONTINENCE: TVT-O}

Objective: We presented our experience in the application of Tvt-O, a surgical a new technic for SUI.

Method: Between April and December 2004, 96 patients underwent Tvt-O surgery, in Uroginecology and Vaginal Surgery Unit of the Gynecology and Obstetrics Department, Las Condes Clinic. Median age was 54 years old, weight 65 kgs. Urodinamia test: SUI II 80 cases, SUI III 4, SUI 0 in 3, and mixed urinary incontinence 9. Gynecare TVT Obturator System technique was used, Ethicon Johnson \& Johnson. It has three specific instruments: helical passers, plastic tubes fixed to mesh of prolene, and a guide for the introduction of the needle.

Results: Media time was 7 minutes (4 to 15). Other gynecological surgery was associated in 77 (80\%). Complications did not appear during the surgical act. No injury to bladder and urethra was observed. We observed in immediate postoperative period only one complication (1.04\%). It was urinary retention. One complication appeared (1.04\%) in remote postoperative period, corresponding polypropylene tape exposition in the anterior vaginal wall. SUI solution was obtained in $100 \%$ of patients. The medium time of observation is 6 months, 51 cases have more than 6 months of observation.

Discussion: Tvt-O is a surgical SUI correction technique with promissory results. Like the other types of TOT, their effectiveness will have in the long term to be evaluated. 
A través de los años, se han descrito una variedad de técnicas quirúrgicas para el tratamiento de la incontinencia de orina de esfuerzo (IOE). Lejanos parecen los tiempos en que se sugerían las llamadas suturas de plicatura, como las descritas por Kelly (1913) ${ }^{1}$, Stockel (1921) ${ }^{2}$ y Marion (1935) ${ }^{3}$. Hasta llegar a la colposuspensión suprapúbica descrita en 1949 por Marshall, Marchetti y $\mathrm{Krant}^{4}$, con sus múltiples modificaciones, hasta llegar a la operación de Burch ${ }^{5,6}$. Esta última fue considerada por muchos años como el gold standard de la cirugía de IOE. Sin embargo en los últimos años han tenido un notable desarrollo las técnicas de corrección con cinta a la zona media uretral. En 1996 Ulmsten describe el TVT ${ }^{7,8}$, una cirugía minimamente invasiva, pero con la desventaja de requerir cistoscopia intraoperatoria, y no exenta de riesgos como lesión vesical, vías urinarias e intestinal, entre otras ${ }^{9-12}$. En el 2001 buscando una mayor simplificación de la técnica, Delorme describe la técnica de TOT (trans-obturatortape) ${ }^{13-15}$. Esta técnica presenta como principal ventaja la posición de la cinta más anatómica que el TVT, las agujas no pasan por el espacio retropúbico, menos sangrado, ausencia de incisiones abdominales, disminución del riesgo de injuria vesical e intestinal, y no requiere cistoscopia. Constituyéndose como una cirugía de mayor facilidad y rapidez en su ejecución, respecto a TVT ${ }^{16}$.

Durante el 2003 Jean de Leval en un intento por simplificar aún más la técnica, describe el TVT- $\mathrm{O}^{17}$.

A continuación presentamos nuestra experiencia en la aplicación de TVT-O, en la corrección quirúrgica de la IOE.

\section{PACIENTE Y MÉTODO}

Entre abril y diciembre del 2004, se realizaron 96 TVT-O a pacientes de la Unidad de Uroginecología y Cirugía Vaginal, del Departamento de Ginecologia y Obstetricia de la Clínica Las Condes.

Se confeccionó una base de datos para registrar prospectivamente a cada paciente.

La mediana de edad fue de 54 años (rango 38 a 74), peso $65 \mathrm{~kg}$ (53 a 82), IMC 27,5 (23 a 33), y paridad vaginal 2,6 (0 a 5).

El test de urodinamia demostró IOE grado II en 80 casos, IOE grado III en 4, IOE grado 0 en 3 , e IOM (Incontinencia de orina mixta) en 9.
Instrumentos quirúrgicos específicos: Se utilizó el Sistema Gynecare TVT Obturador de Ethicon, Johnson \& Johnson. Este consiste en tres instrumentos específicos: un pasador de aguja helicoidal, un tubo plástico fijo a la malla de prolene, y una guía facilitadora para la introducción de la aguja. (Fig. 1).

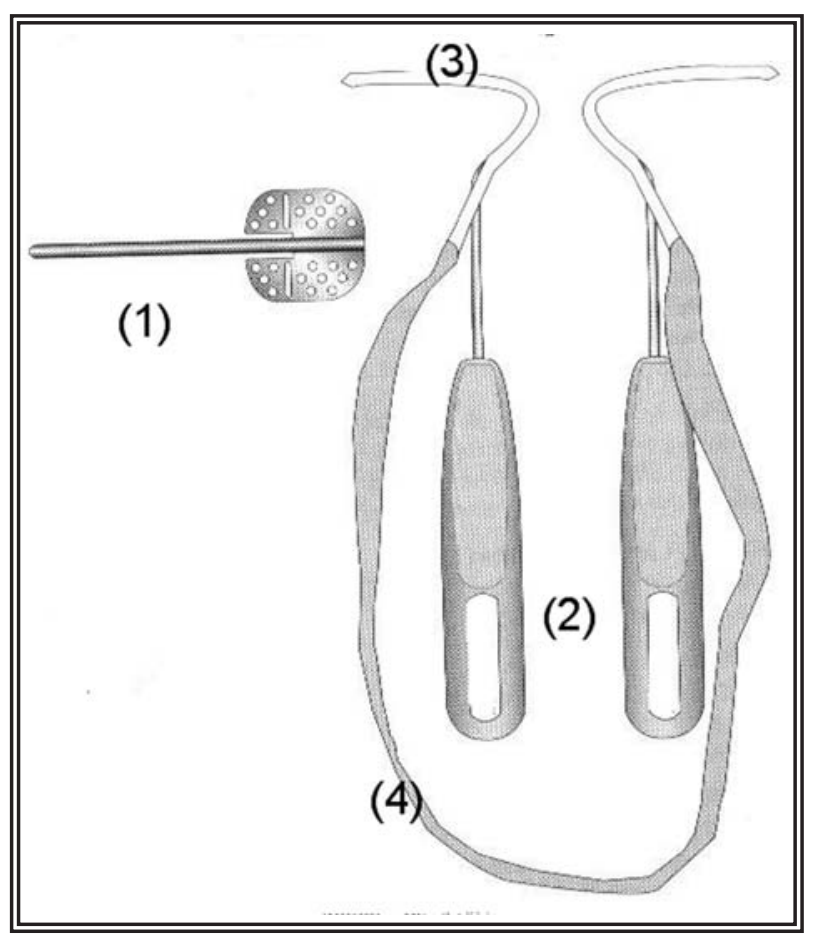

FIGURA 1. Sistema Gynecare TVT Obturador de Ethicon, Johnson \& Johnson. (1) Guia facilitadora para la introducción de la aguja, (2) pasador de aguja helicoidal, (3) tubo plástico fijo a la (4) malla de prolene.

Pasador con aguja helicoidal: consiste en un par de instrumentos, uno para cada lado. Formado por un mango para asir, y una aguja en su extremo. Esta última posee una curvatura helicoidal que se abre hacia externo.

Tubo plástico: se fija al extremo helicoidal de la aguja, y posee fija en su parte más distal la malla de prolene (polipropileno), que a su vez se encuentra protegida por una envoltura plástica.

Guía: facilita una introducción y pasaje seguro de la aguja con el tubo y la malla. Posee dos alas en su extremo proximal, que son superficie para manipular la guía, además de evitar su rotación involuntaria.

Técnica quirúrgica: El procedimiento quirúrgico se realizó bajo anestesia raquídea, y en los 
casos en que se asoció alguna cirugía laparoscópica, anestesia general.

Se utilizó antibiótico profilaxis con cefazolina 2 g endovenoso, en una sola dosis una hora previa a la cirugía.

Se colocó a la paciente en la camilla operatoria en posición ginecológica. Se limpió la superficie operatoria con povidona yodada. Se colocó una Sonda Foley de 16 fr. Luego se siguió con la técnica quirúrgica específica:

Paso 1: Se marcaron los puntos de salida de las agujas. Para ello se traza una línea horizontal a la altura del meato urinario. Otra linea paralela y a 2 centímetros por sobre la anterior. Finalmente se localizará el punto de salida en esta última línea, a $2 \mathrm{~cm}$ por fuera del pliegue del muslo. La incisión fue de sólo $3 \mathrm{~mm}$. Esto se realiza a ambos lados (Fig. 2).

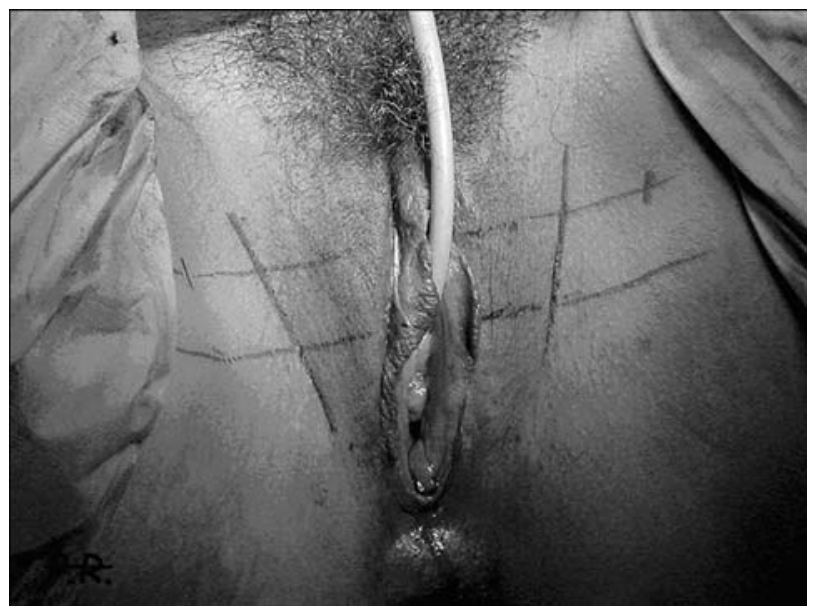

FIGURA 2. Paso 1: Se marcan los puntos de salida de las agujas. Primera linea horizontal a la altura del meato urinario. Segunda línea a 2 centimetros por sobre la anterior. Punto de salida en esta última línea, a $2 \mathrm{cms}$ por fuera del pliegue del muslo.

Paso 2: Se abre la mucosa de la pared vaginal anterior a un centímetro bajo el meato urinario. La incisión es de un centímetro. Se diseca lateralmente y a ambos lados de la zona media uretral con tijera fina, con un ángulo de 45 grados a la línea media, orientándose inmediatamente por debajo del plano horizontal de la mucosa, y en dirección a los puntos de salida descritos en el paso 1. Cuando se encuentra el borde superior de la rama ósea isquiopubiana, se perfora la membrana obturatriz con la punta de la tijera (Fig. 3).

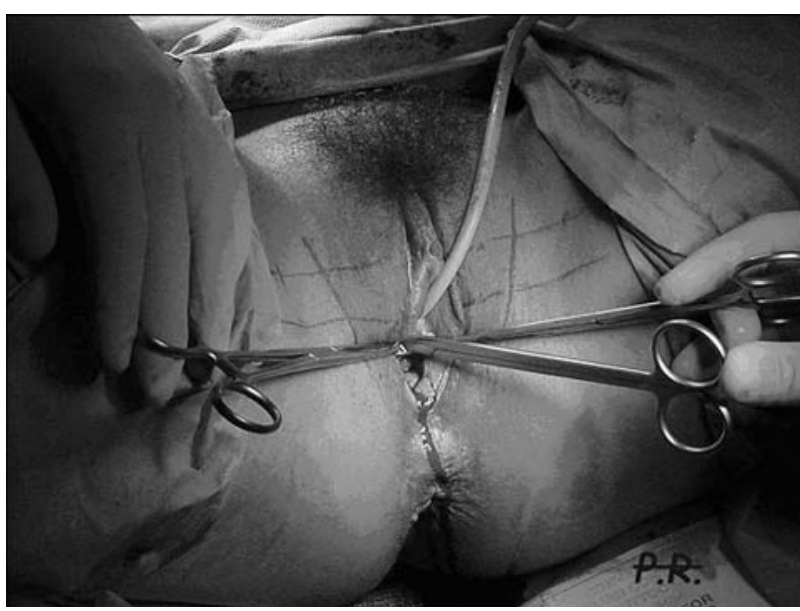

FIGURA 3. Paso 2: Abierta la mucosa de la pared vaginal anterior a un centímetro bajo el meato urinario (incisión es de un centimetro). Se diseca lateralmente y a ambos lados de la zona media uretral con tijera fina, con un ángulo de 45 grados a la línea media, y en dirección a los puntos de salida descritos en el paso 1.

Paso 3: Se introduce la guía, pasando su extremo distal por la apertura de la membrana obturatriz.

Paso 4: Se pasa la aguja armada con el tubo y la malla, apoyándola y deslizándola en el carril que entrega la guía. Para ello se desliza siguiendo la curvatura helicoidal natural de la aguja, y orientándola hacia el punto de salida. Luego se retira la guía (Fig. 4).

Paso 5: Se toma el extremo del tubo plástico que ha salido por la apertura de la piel, con una pinza de Kelly. Y se retira la aguja siguiendo su curvatura, en el paso inverso a su introducción (Fig. 5).

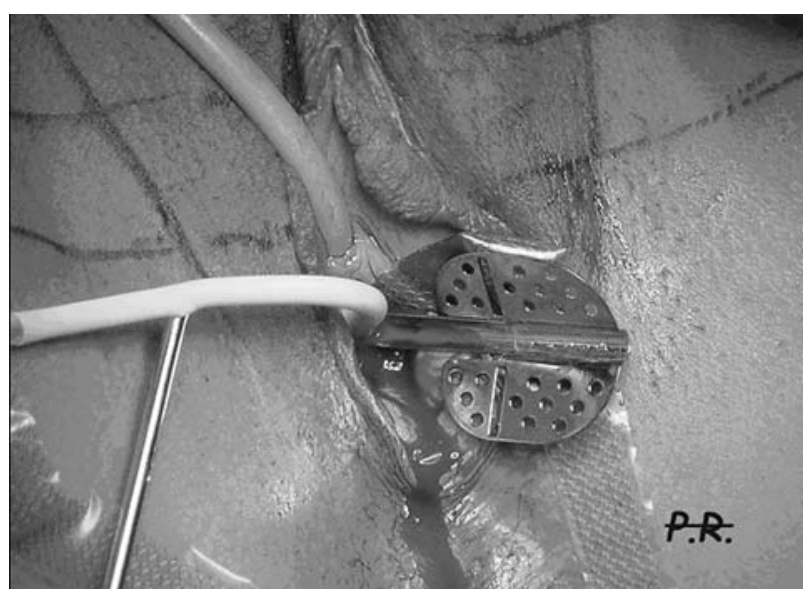

FIGURA 4. Paso 4: Paso de la aguja armada con el tubo y la malla, apoyándola y deslizándola en el carril que entrega la guia. Se desliza siguiendo la curvatura helicoidal natural de la aguja, y orientándola hacia el punto de salida. Luego se retira la guia. 


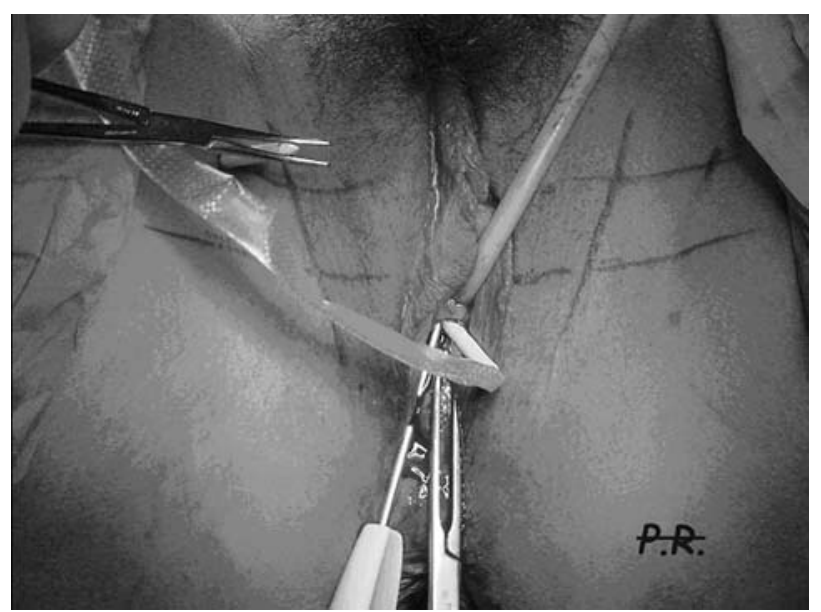

FIGURA 5. Paso 5: Se toma el extremo del tubo plástico que ha salido por la apertura de la piel, con una pinza de Kelly. Luego se retira la aguja siguiendo su curvatura, paso inverso a su introducción.

Paso 6: Se tracciona el tubo plástico a través de la piel hasta que aparezca la malla (Fig. 6). Se repite la misma técnica en el lado contralateral.

Paso 7: Se ajusta la malla y se remueve la cubierta plástica que la envuelve. Para ajustar la tensión, se introduce una tijera entre la uretra y la malla mientras se retira la envoltura plástica (Fig. 7). Esta última queda libre de tensión, una vez cortada la unión al tubo plástico, permitiendo su retiro por tracción a través de la piel a cada lado, ya que se encuentra separada en su parte media. Luego se corta la cinta a nivel subcutáneo, y se afronta la piel (no es necesario suturar). Finalmente se sutura la mucosa vaginal con polipropileno 3-0.

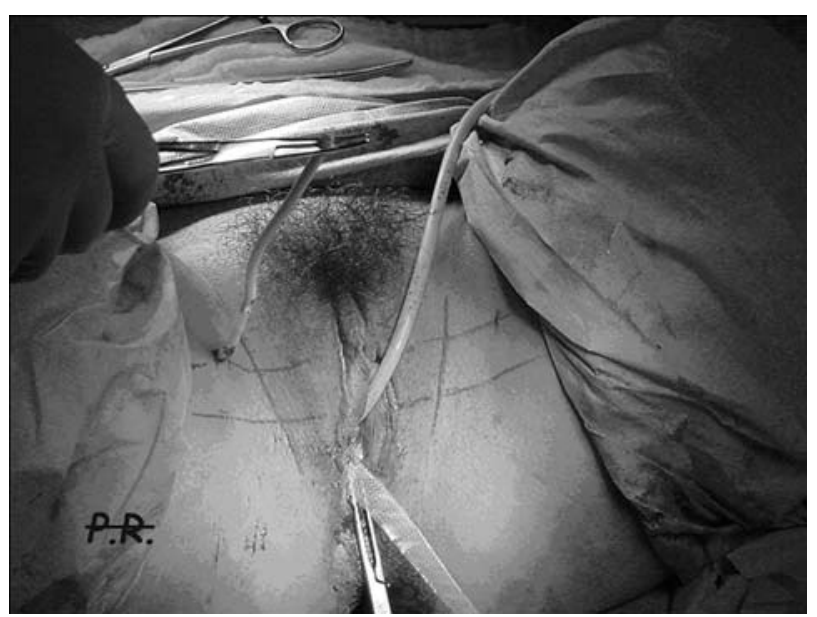

FIGURA 6. Paso 6: Se tracciona el tubo plástico a través de la piel hasta que aparezca la malla.

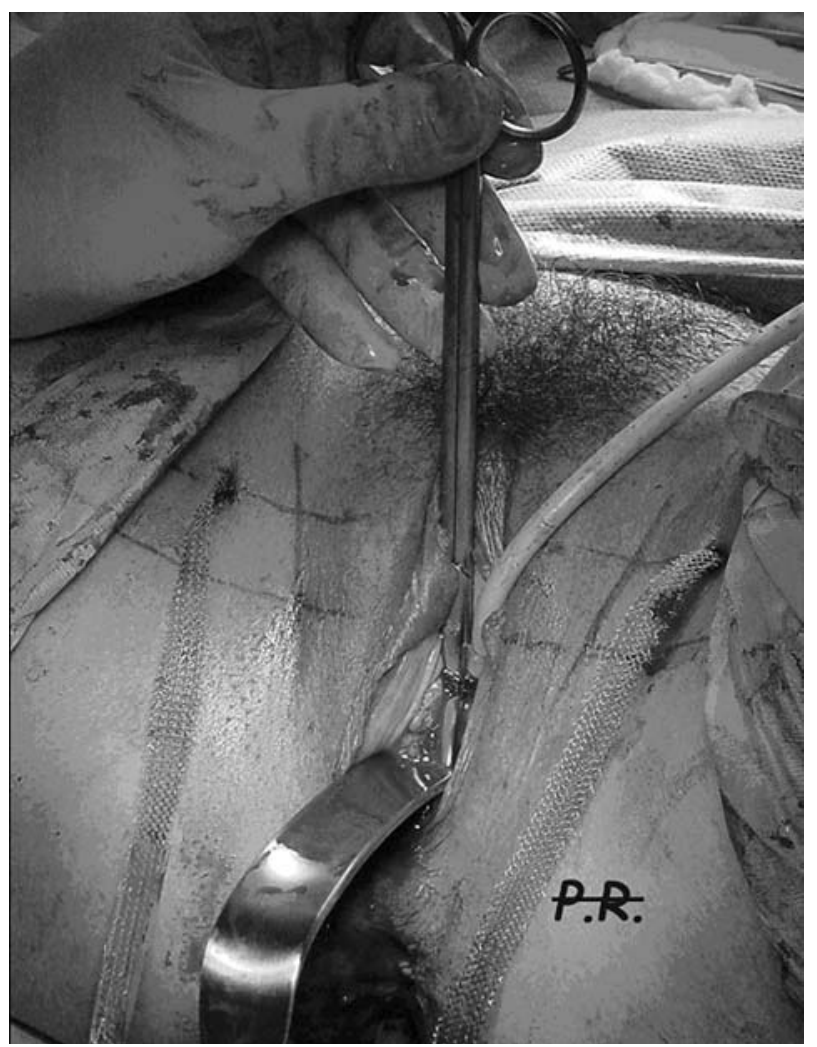

FIGURA 7. Paso 7: Se ajusta la malla y se remueve la cubierta plástica que la envuelve. Para ajustar la tensión, se introduce una tijera entre la uretra y la malla.

En los casos en que asoció otra cirugía ginecológica, se realizó en todos los casos en un tiempo posterior al TVT-O.

La sonda Foley se retiró una vez terminada la intervención de TVT-O sólo, y al completar 24 horas de postoperatorio en los casos con cirugía complementaria.

La analgesia se realizó con Rofecoxib $50 \mathrm{mg}$ vía oral, una hora antes de la cirugía, repitiéndose a las 24 horas posteriores. Este esquema fue reemplazado por Valdecoxib $40 \mathrm{mg}$ en igual indicación, a partir de noviembre del 2004.

A todas las pacientes se les explicó previamente, en qué consistía el TVT-O, y en los casos correspondientes, el procedimiento quirúrgico concomitante a realizar. Todas firmaron un consentimiento informado.

Se controló en consulta ambulatoria entre los 7 y 14 días, al mes, a los 3 y 6 meses.

\section{RESULTADOS}

La mediana del tiempo operatorio del TVT-O fue de 7 minutos, con rango entre 4 y 15 minutos. 
Se asoció alguna cirugía ginecológica a 77 (80\%) de las 96 pacientes. Estas fueron: histerectomía vaginal, histerectomía laparoscópica, colpoperineoplastia anterior y posterior, esterilización tubaria laparoscópica, y vulvectomía parcial. En algunos casos se asoció hasta 2 cirugías.

No se presentaron complicaciones durante el acto quirúrgico. No se observó daño de vejiga ni vías urinarias. $\mathrm{El}$ sangrado intraoperatorio fue escaso (menor a $80 \mathrm{ml}$ ).

Durante el postoperatorio inmediato se presentó sólo una complicación (1 de 96, 1,04\%) Correspondiendo a una retención urinaria, producto de sobrecorrección. Fue necesario realizar una corrección en pabellón a las 24 horas de la primera cirugía. Se retiraron las suturas de la mucosa de la pared vaginal anterior $(1 \mathrm{~cm})$. Y se reajustó la cinta realizando tracción por medio de una pinza de Kelly entre la uretra y la malla de polipropileno. Este caso correspondía a TVT-O asociado a colpoperineoplastia posterior.

En ningún caso se presentó hematoma ni infección de la zona operatoria.

No fue necesario agregar farmacoterapia adicional al esquema para dolor postoperatorio planteado inicialmente. Ya que en todos los casos se logró un adecuado control, expresado por la paciente.

El alta a domicilio se dio en los casos de TVTO sin asociación a otra cirugía, después de observar la segunda micción espontánea. Con una mediana de 12 horas. En los casos en que se asoció colpoperineoplastia anterior o posterior, se dio alta a las 24 horas. En los casos de asociación de histerectomía, a las 48 horas.

Durante el postoperatorio tardío se presentó una complicación (1 de 96, 1,04\%), correspondiendo a un caso de exposición de la cinta de polipropileno en la mucosa de la pared vaginal anterior. Correspondiendo a $1 \mathrm{~cm}^{2}$ de erosión. Fue necesario seccionar la cinta en pabellón, y no presentó nueva complicación. En este caso se había realizado TVT-O asociado a colpoperineoplastia anterior y posterior.

No se presentaron casos de fístulas.

Se obtuvo solución de la incontinencia de orina de esfuerzo en las 96 pacientes (100\%), incluidas las pacientes con complicación del postoperatorio inmediato y tardío.
En las pacientes con IOE (87 casos), se presentó incontinencia de urgencia de novo en 2 casos (2,3\%). Ambos con resolución espontánea, a los 30 días y a los 45 días respectivamente.

En los casos con incontinencia de orina mixta (9 casos), se mantuvo la urgencia miccional sólo en 1 paciente. Se trató con tolterodina, con respuesta completa.

Todas fueron controladas según protocolo, el cual se continúa cumpliendo a la fecha en muchas de ellas. La mediana de seguimiento para los 96 casos es de 6 meses, destacando 51 casos con seguimiento mayor a 6 meses. Los casos de seguimiento mayor han completado 11 meses.

\section{DISCUSIÓN}

Muchos sostienen que el TVT debe considerarse como el gold standard de la corrección quirúrgica de la incontinencia de orina de esfuer$\mathrm{zO}^{18}$. Con cifras de hasta $81 \%$ de curación completa y $16 \%$ parcial $^{19}$. De lo que no debiera existir duda alguna, es que las técnicas de cinta de polipropileno localizada en la uretra media, debe considerarse como la cirugía de elección en la $\mathrm{IOE}^{20,21}$. El gold standard futuro será aquella cirugía que logre una continencia completa, sin morbilidad de importancia.

El TVT-O se acerca más aún al concepto de cirugía minimamente invasiva, que sus antecesores TVT y TOT. La disección de la pared vaginal anterior es sólo de un centímetro; la disección del espacio parauretral es con tijera fina; se utiliza una guía para facilitar la introducción de la aguja; el paso de las agujas se realiza una sola vez y se retira del interior de un tubo plástico que la protege; y no se requiere cistoscopia.

Se trata de una técnica quirúrgica que al poseer una guía para la introducción de las agujas, evitaría posibles daños de vejiga y vías urinarias. Una revisión realizada en 76 casos de TVT realizados en nuestra Unidad de Uroginecología entre octubre del 2001 y marzo 2004, demostró un 5\% de perforación vesical, sin necesidad de intervención quirúrgica adicional para su corrección (TVT en asociación a otras cirugías ginecológicas durante el mismo acto quirúrgico, y sus complicaciones. Sometido a publicación).

Las complicaciones que se observan en nuestra experiencia son escasas, y de fácil solución. 
Es una cirugía de técnica sencilla, y que se realiza en tiempo reducido. Se puede asociar a otras cirugias ginecológicas, permitiendo una solución más integral a las pacientes durante un mismo acto quirúrgico. Con un postoperatorio que requiere escasos cuidados, debido al escaso dolor, retiro precoz de la sonda Foley, y alta hospitalaria temprana.

Importante resulta mencionar que esta técnica permite conservar indemne un puente mucoso suburetral. Ya que se realiza una pequeña incisión de 1 centímetro para ingresar, y en caso de necesidad de plastia de pared anterior, una segunda incisión apartada de la anterior, dejando esa zona indemne. Por otro lado al ingresar por una pequeña incisión, evita la migración de la cinta posteriormente.

Por otro lado, el análisis preliminar de los resultados de seguimiento, a casi un año de iniciada esta cirugía en nuestra unidad, permite comunicar curación de la IOE en todos los casos operados. Sin embargo en futuras publicaciones, estaremos en propiedad de informar las tasas de curación a largo plazo.

En conclusión, nuestra experiencia nos evidencia que TVT-O es una técnica quirúrgica para el tratamiento de la IOE, con resultados a la fecha actual muy promisorios. Al igual que para los otros tipos de TOT, su eficacia deberá ser evaluada a largo plazo ${ }^{22}$.

\section{REFERENCIAS}

1. Kelly HA. Incontinente of urine in women. Urol Cutan Rev 1913;17:291.

2. Stoeckel W. Doe Therapie der incontinentia urinae bei traumatischer Schadigung der Sphinktermuskulatur. Zbl Gynakol 1921;45:17.

3. Marion G. Traité d'Urologie. Paris:Masson, 1935.

4. Marshall VF, Marchetti AA, Krantz KE. The correction of stress incontinence by simple vesicourethral suspension. Surg Gynecol Obstet 1949;88:509.

5. Burch JC. Urethrovaginal fixation to Cooper's ligament for correction of stress incontinence, cystocele, and prolapse. Am J Obstet Gynecol 1961;81:281.

6. Burch JC. Cooper's ligament urethrovesical suspension for stress incontinence. Nine years' experience - results, complications, techniques. 1968. Am J Obstet Gynecol 2002; 187(2):512.

7. Ulmsten U, Henriksson L, Johnson P, Varhos G. An ambulatory surgical procedure under local anesthesia for treatment of female urinary incontinence. Int Urogynecol J Pelvic Floor Dysfunct 1996;7(2):81-85.

8. Rezapour M, Ulmsten U. Tension-free vaginal tape (TVT) in women with mixed urinary incontinence -a long-term follow-up. Int J Urogyneco J Pelvic Floor Dysfunct 2001;12 suppl:15-18.
9. Ulmsten U, Falconer C, Johnson P, Jomaa M, Lanner L, Nilsson CG, et al. A multicenter study of tension-free vaginal tape (TVT) for surgical treatment of stress urinary incontinence. Int Urogynecol J Pelvic Floor Dysfunct 1998; 9(4): 210-213.

10. Allahdin S, Mckinley C, Mahmood TA, Lyth D. Tensionfree vaginal tape: 162 cases in a district general hospital. J Obstet Gynaecol 2004;24(5):539-541.

11. Abouassaly R, Steinberg JR, Lemieux M, Marois C, Gilchrist LI, Bourque JL, et al. Complications of tensionfree vaginal tape surgery: a multi-institutional review. BJU Int 2004; 94(1):110-113.

12. Jimenez CJ, Hualde AA, Gonzalez DE Garibay AS, Pinos PM, Jimenez AJ, et al. TVT: three years of experience Actas Urol Esp 2004;28(1):13-20.

13. Delorme E. Transobturator urethral suspension: miniinvasive procedure in the treatment of stress urinary incontinence in women Prog Urol. 2001;11(6):1306-1313.

14. Delorme E, Droupy S, De Tayrac R, Delmas V. Transobturator tape (Uratape). A new minimally invasive method in the treatment of urinary incontinence in women Prog Urol 2003;13(4):656-659.

15. Costa P, Grise P, Droupy S, Monneins F, Assenmacher C, Ballanger P,et al. Surgical treatment of female stress urinary incontinence with a trans-obturator-tape (T.O.T.) Uratape: short term results of a prospective multicentric study. Eur Urol 2004;46(1):102-106.

16. Mellier G, Benayed B, Bretones S, Pasquier JC. Suburethral tape via the obturator route: is the TOT a simplification of the TVT? Int Urogynecol $\mathrm{J}$ Pelvic Floor Dysfunct 2004;15(4):227-232.

17. De Leval J. Novel surgical technique for the treatment of female stress urinary incontinence: transobturator vaginal tape inside-out. Eur Urol 2003;44(6):724-730.

18. Debodinance P, Delporte P, Engrand JB, Boulogne M. Tension-free vaginal tape (TVT) in the treatment of urinary stress incontinence: 3 years experience involving 256 operations. Eur J Obstet Gynecol Reprod Biol. 2002;105(1): 49-58.

19. Nilsson GC, Rezapour M, Falconer C. 7 year follow-up on the Tension-free Vaginal Tape (TVT) procedure; International Urogynecology Journal, IUGA Abstract 116 (89): October 2003.

20. Rechberger T, Wrobel A, Adamiak A, Skomra D, Korobowicz E, Tomaszewski J, et al. Tissue reaction to polypropylene mono-or multi-filament tapes used in surgical techniques of stress urinary incontinence treatment Ginekol Pol 2003;74(9):1008-1013.

21. Bemelmans BL, Chapple CR. Are slings now the gold standard treatment for the management of female urinary stress incontinence and if so which technique? Curr Opin Urol. 2003; 13(4):301-307.

22. Costa P, Delmas V. Trans-obturator-tape procedure "inside out or outside in": current concepts and evidence base. Curr Opin Urol 2004;14(6):313-315.

Dr. P. Ricci Arriola

Unidad de Uroginecología y Cirugía Vaginal,

Clínica Las Condes

Lo Fontecilla 441, Las Condes,

Santiago, Chile.

(Trabajo recibido el 31 de enero 2005) 